\title{
A STUDY OF NONLINEAR DYNMAICS OF MEMS INERTIAL SENSOR UNDER OVERLOAD CONDITIONS
}

\author{
David Lin, Todd Miller, and Krithivasan Suryanarayanan \\ Freescale Semiconductor, Inc., Tempe, Arizona, USA
}

\begin{abstract}
This paper reports the study results on the nonlinear dynamics of a z-axis MEMS capacitive acceleration sensor in the context of overload conditions. Two dominant sources of nonlinearity are discussed - nonlinear squeeze-film damping and higher order eigenmodes of the mechanical structure. The nonlinear damping results were obtained by solving large displacement Reynolds equation and FEA simulation. Reduced order model is employed to incorporate the higher order modes into microsystem model for crash analysis. It is shown that the microsystem model can accurately simulate the crash overload event when properly taking into accounts these two sources of nonlinearity.
\end{abstract}

\section{INTRODUCTION}

The dynamic performance of microelectromechanical system (MEMS) inertial sensors is a critical criterion for success in automotive safety application [1]. Typically the device is designed to operate within a specified range. Acceleration beyond the range of the device (overload) can generate a DC shift at the output of the device that is dependent upon the overload frequency and amplitude. In particular, the high frequency, high amplitude stimulus during crash event often causes nonlinear dynamic behavior of MEMS sensors, which manifests itself as DC shift at the output, resulting in erroneous airbag deployment.

In this work, two dominant sources of nonlinearity in MEMS dynamics are studied in the context of overload conditions nonlinear squeeze-film damping and higher order eigenmodes of the mechanical structure.

As an example to illustrate our methodology and computational results, a teeter totter style Z-axis MEMS capacitive acceleration sensor (Fig. 1) was designed and fabricated. This transducer was designed to operate at a full-scale acceleration range of $250 \mathrm{~g}$ but was tested at overload condition of more than $4000 \mathrm{~g}$ acceleration stimulus. The MEMS sensor was fabricated by a two-layer polysilicon surface micromachining process and sealed in a hermetic cavity with a controlled pressure. Under large Z-axis acceleration, the proof mass will have significant rotation around the pivot point. Such a large displacement can cause unexpected nonlinear response which is the focus of this study.

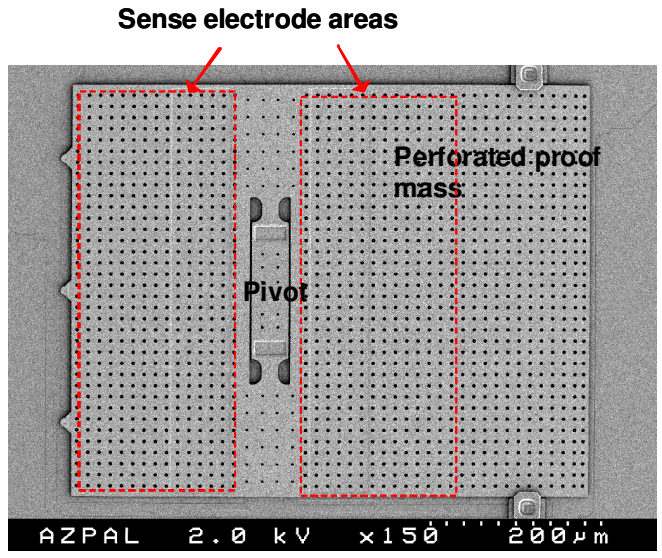

Figure 1: SEM picture of the teeter totter MEMS acceleration transducer for crash sensing application.

\section{NONLINEAR SQUEEZE-FILM DAMPING Analytical Solutions}

Reynolds equation known from lubrication theory is often used to model the squeeze film damping for plate-like microstructure moving towards a fixed wall [2] [3]. Reynolds squeeze film equation assumes a continuous fluid flow regime, but can be conveniently adjusted to expand into the results of transition or molecular regime by introducing the effective dynamic viscosity proposed in [4]. This holds true for MEMS acceleration sensors which are typically encapsulated in a non-vacuum environment in order to have over-damping characteristics. However, due to the large displacement of MEMS structure under the overload conditions (Fig. 2), the microstructure will demonstrate nonlinear damping behavior. When the proof mass moves from the nominal position towards the substrate, damping will increase with the reduction of film gap. When the proof mass is moving away from substrate, damping will decrease.
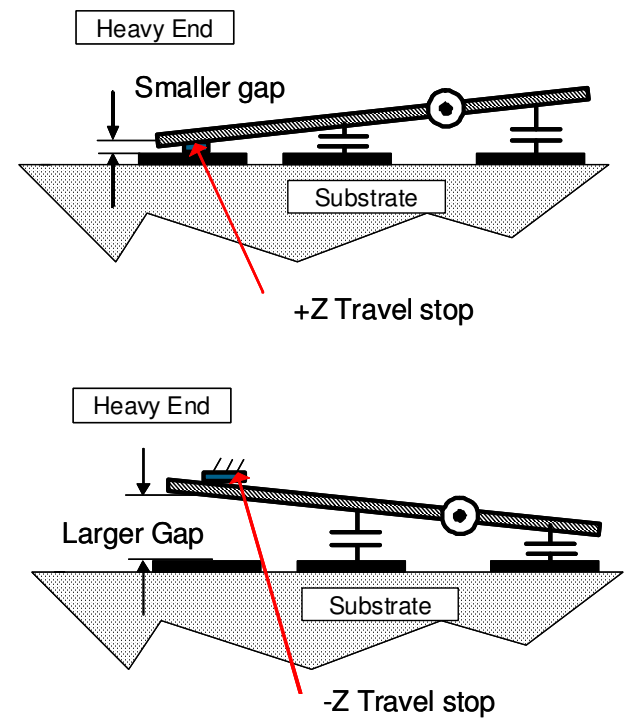

Figure 2: Non-linear squeeze film damping caused by the large displacement of the proof mass.

In the large displacement case, the actual gap change is in the same order of magnitude of the initial film gap. The linearized Reynolds equation is no longer valid for squeeze-film damping. Instead, the nonlinear Reynolds equation (Eq. 1) has to be used:

$$
\begin{aligned}
& \frac{\partial}{\partial x}\left(\frac{P H^{3}}{\mu} \frac{\partial P}{\partial x}\right)+\frac{\partial}{\partial y}\left(\frac{P H^{3}}{\mu} \frac{\partial P}{\partial y}\right)=12 \frac{\partial(P H)}{\partial t} \\
& H=h_{0}+h(t, x, y), \\
& P=p_{0}+p(t, x, y), \\
& \mu \text { - viscosity } \quad p_{0} \text { - cavity pressure } \\
& h_{0} \text { - film gap } \quad p(t, x, y) \text { - pressure change } \\
& h(t, x, y) \text { - gap change }
\end{aligned}
$$


In the simplest cases where no damping from the perforated holes is assumed, modal transformation technique was applied to separate the spatial variables $(x, y)$ and time variable $(t)$. The squeeze film gap variation and the pressure variation can be written as:

$$
\begin{aligned}
& h(t, x, y)=q(t) f(x, y) \\
& p(t, x, y)=\sum_{n, m=1}^{\infty} p_{m n}(t) \sin \frac{m \pi x}{L} \sin \frac{n \pi y}{W}
\end{aligned}
$$

where $f(x, y)$ is the eigenvector of the first mode of the teeter totter transducer and $q(t)$ is the modal time function. A lower-order equation written in the Fourier coefficients of the pressure distributions $\left(P_{m n}\right)$ can be obtained by substituting Eq. 2 and 3 into Eq. 1 and applying a harmonic excitation:

$$
q(t)=\sin (\omega t)
$$

The resulted equation has nonlinear terms and is therefore solved by Runge-Kutta Method. Fig. 3 shows the solution results for a simplified model of the MEMS structure under $20 \mathrm{KHz} 7500 \mathrm{~g}$ sinusoidal acceleration. The result is presented in the frequency domain. Compared with the constant damping case, the higher order harmonics caused by the nonlinear squeeze film damping can clearly be seen.
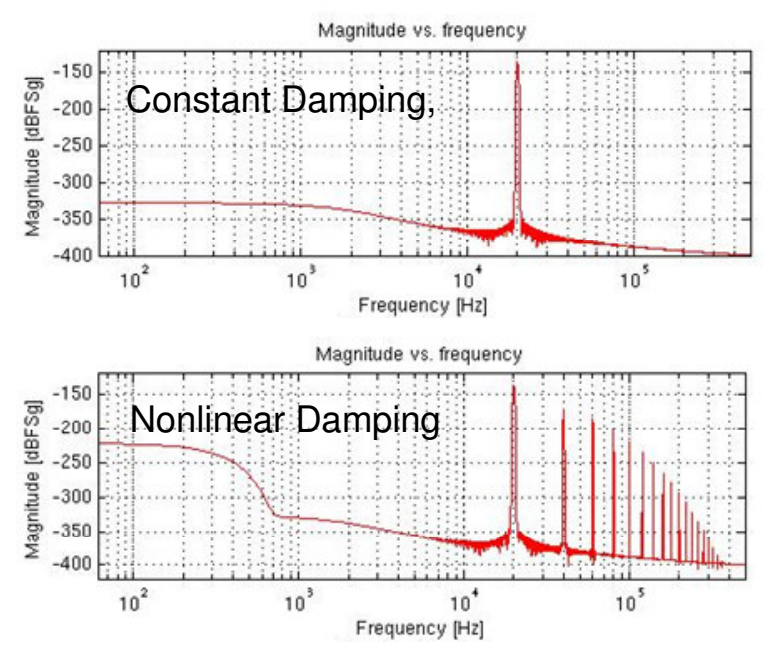

Figure 3: Frequency domain modeling results of MEMS structure subject to $20 \mathrm{KHz} 7500 \mathrm{~g}$ sinusoidal acceleration.

\section{Finite Element Analysis (FEA)}

For complicated structures with inhomogeneously distributed perforations, analytical solution has limits. FEA modeling was instead utilized to study the non-linear squeeze film damping behavior. The squeeze film damping was simulated based on the fluid-structure interaction by using a commercial FEA tool. Both structural and thin-film fluid models were built. Additional flow rate elements were introduced to model the flow resistance through the perforations [5]. Modal analysis on the structural domain was performed to determine the resonant frequencies and extract the eigenvectors for each desired mode. Harmonic analysis was performed on the thin-film elements to reveal the pressure distribution for each considered mode. Modal pressures are projected onto the particular vibrations modes to obtain generalized modal squeeze film damping forces.. This procedure was repeated for various film gaps to extract the damping ratio as a function of gap change. Figure 4 shows the FEA model and the simulated pressure distribution within the proof mass region for the first eigenmode of the teeter totter $\mathrm{z}$-axis transducer.
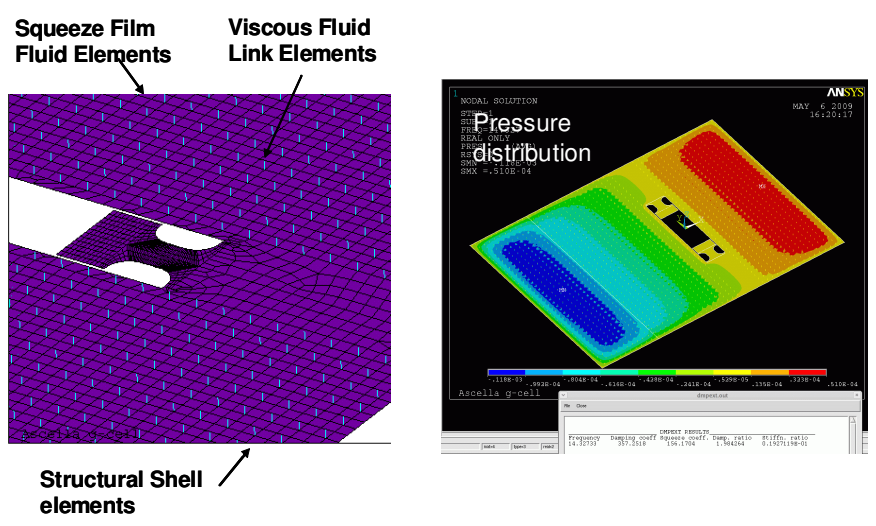

Figure 4: FEA simulation of the nonlinear squeeze film damping of the teeter totter MEMS sensor.

Characterization by using a white light interferometer was also performed to verify the FEA simulation results. The MEMS sensor was mounted inside a chamber where the pressure can be regulated to match the actual micro-cavity pressure from wafer bonding. A high DC voltage bias and a small AC signal were applied between the movable plate and the stationary electrodes. The DC voltage is used to create electrostatic force to move the proof mass around the pivot point, thus to change the film gap between the proof mass and substrate. The AC signal is to actuate the proof mass dynamically so that the resonant frequencies and damping factor can be extracted. An exemplary white-light interferometer image of the deformed microstructure and the test setup are shown in Fig. 5. By varying the DC control voltage, the damping ratio change with respect to gap change can be obtained experimentally.
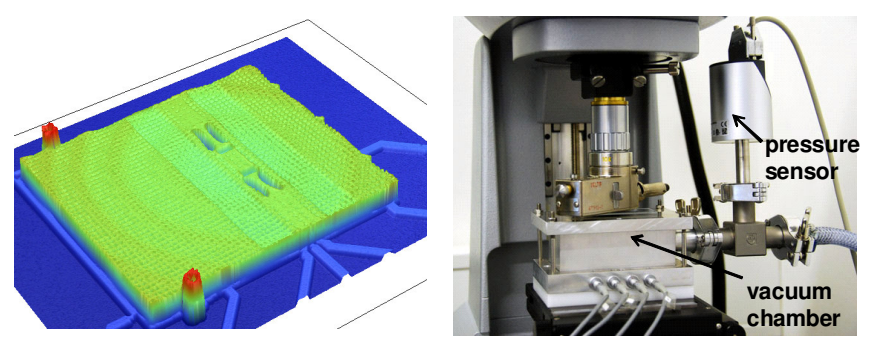

Figure 5: Characterization of the squeeze film damping by white light interferometer.

Both simulation and characterization results of the squeezefilm damping are presented in Fig. 6. The increase of squeeze-film due to gap closing can clearly be seen on both the simulation and experiment. A reasonable good agreement is also found between them. Note the characterization can only be performed in a smaller range of gap change because of the pull-in phenomenon of moving plate. The simulation results in general deviate more from test data at larger film gap. This is due to the additional impact of fluidic transition regions at plate boundaries and hole apertures, which is considered in simulation as an additional correction factor but with limited accuracy.

Fig. 7 further shows the time domain simulation results of the MEMS accelerometer when being subject to $10 \mathrm{KHz} 7500 \mathrm{~g}$ 
acceleration. Simulation reveals that the transducer output can deviate by more than $20 \%$ if the nonlinear squeeze film damping is not properly considered. This shows the significance of including the nonlinear squeeze film effect in overload investigation.

Nonlinear Damping of Teeter Totter Transducer

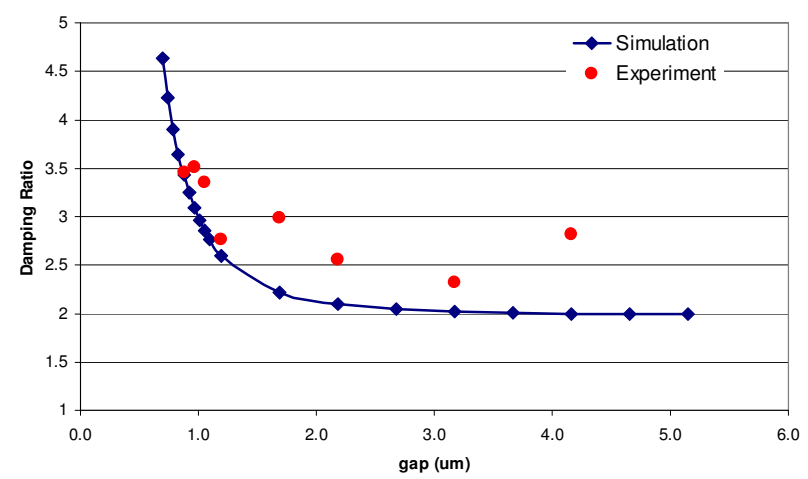

Figure 6: Comparison of the FEA results and characterization results.

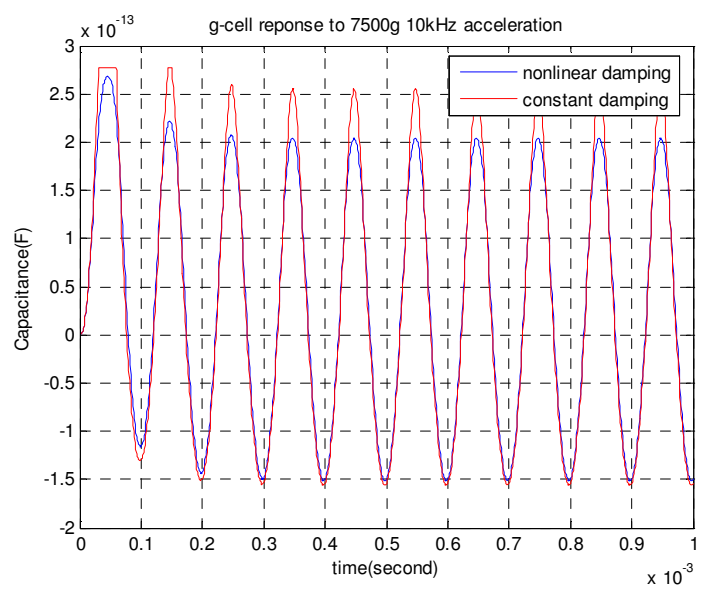

Figure 7: Time domain modeling results of the MEMS structure subject to $10 \mathrm{KHz} 7500 \mathrm{~g}$ acceleration.

\section{HIGHER ORDER EIGENMODES Electrical Nonlinearity}

Mode projection technique has been discussed in the previous section for solving the modal squeeze film damping. This section deals with the other nonlinear effect contributed by the higher order eigenmodes. As shown in Fig. 8, the teeter totter acceleration transducer has other higher frequency eigenmodes in addition to the desired eigenmode (primary mode) for sensing Z-axis acceleration stimulus. Because of the high frequency contents of crash signal and lower damping at higher order modes, these high order eigenmodes can be excited and cause undesirable DC shift.

In mechanical domain, the high order modes do not necessarily create nonlinearity problem because the amount of the proof mass displacement is still small compared with the overall dimension of the structure and stress-stiffening effect is usually very small. This is important in that modal superposition technique can still be applied. However, the capacitance transfer function is a nonlinear transfer function of the proof mass displacement. Often the sensor is designed to have differential architecture such that the nonlinear capacitance output from the primary mode is kept at minimum. However, this generally does not work for higher order modes. The electrical nonlinearity from the higher order modes becomes a significant contributor of nonlinear device output under the large acceleration amplitude and high frequency stimulus, i.e. overload conditions. In other words, the high order eigenmodes of the mechanical structure, when coupled with electrostatics, can produce nonlinear capacitance output.

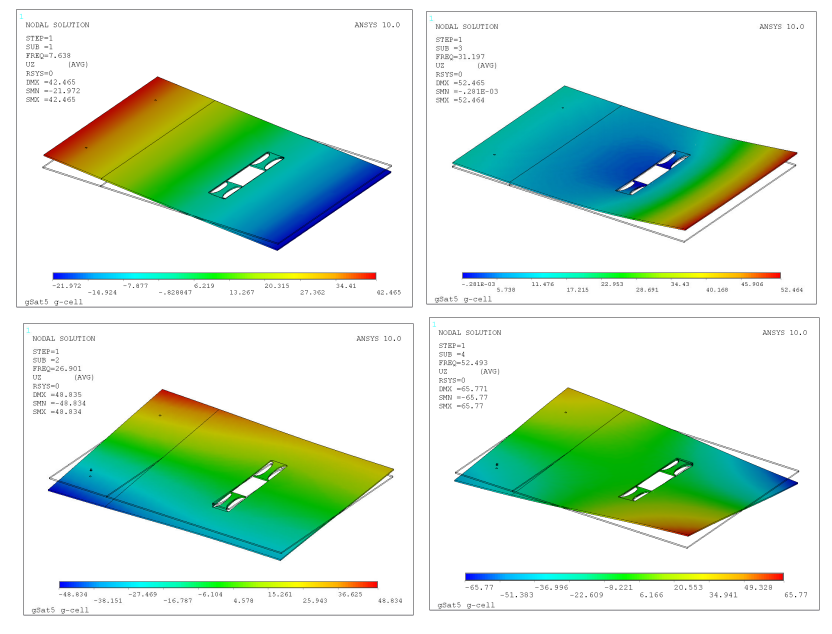

Figure 8: Different eigenmodes of the teeter totter acceleration sensor.

\section{Reduced Order Modeling}

In order to study the contribution of higher order eigenmodes under overload conditions, one must have a high fidelity model which can not only capture the full dynamic behavior of the MEMS device, but also enable fast simulation. Our approach to simulate this behavior was to represent the deformation state of mechanical components by a superposition of finite numbers of eigenmodes. This created a reduced order model (ROM) which enables fast dynamic simulations of linear systems ([6]). Hence, the number of parameters can be reduced from several thousands to a few ten state variables with reasonable accuracy.

ROM has been extended to account for electrostatic-structural interactions in MEMS by introducing multivariable capacitancestroke-functions $\left(C_{r k}\right)$ where stroke is represented by modal amplitudes [7]. The capacitance-stroke relationships are automatically derived from finite element data sampling procedures and subsequent functions fit algorithms. The governing equations for modal amplitudes $q_{i}$ in the mechanical domain become

$$
m_{i} \ddot{q}_{i}+2 \xi_{i} \omega_{i} m_{i} \dot{q}_{i}+m_{i} \omega_{i}^{2} q_{i}=f_{i}+\sum_{r} \frac{\partial C_{r k}}{2 \partial q_{i}}\left(V_{r}-V_{k}\right)^{2}
$$

where $\xi_{i}$ are modal damping ratios, $\omega_{i}$ are eigen-frequencies, $f_{i}$ are load contribution factors, $V_{k}$ and $V_{r}$ electrode voltages.

Figure 9 shows the schematic view of the ROM for the teeter totter MEMS sensor. The natural frequencies and eigenvectors, capacitance functions used in ROM were obtained from FEA while the modal damping factors were obtained from the interferometer measurement. The ROM was implemented in behavioral models and incorporated into microsystem model (next section) so that the nonlinearity of device output caused by the higher order modes can be correctly assessed. 


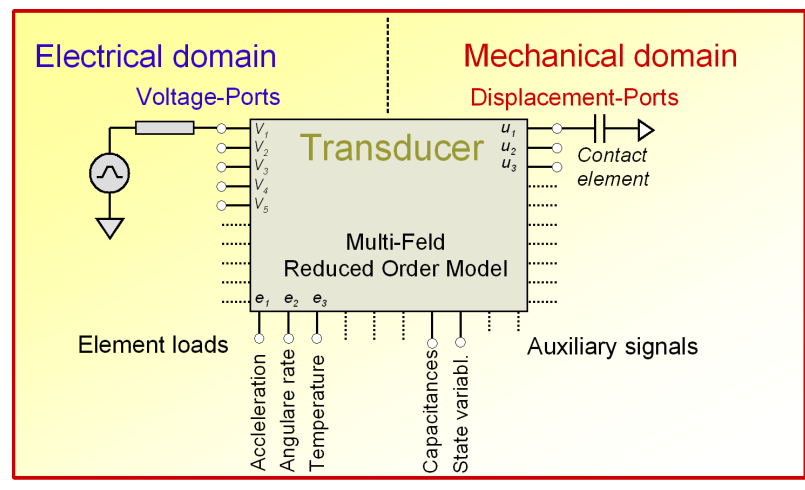

Figure 9: Schematic view of the reduced order sensor model.

\section{MICROSYSTEM INTEGRATION}

Finally, the nonlinear transducer model was incorporated into a microsystem model (Fig. 10). The microsystem model consists of a package model, the MEMS transducer model (with nonlinear damping and higher order modes) and the ASIC signal chain block. System level simulation was performed which took the input from the reference accelerometer and predicted the device output. Experimental verification was also performed using a commercial mechanical shock tester (Fig 11). Fig. 12 shows the good agreement between the modeling and the testing results for the MEMS device subject to $3000 \mathrm{~g}, 2 \mathrm{~ms}$ shock pulse.

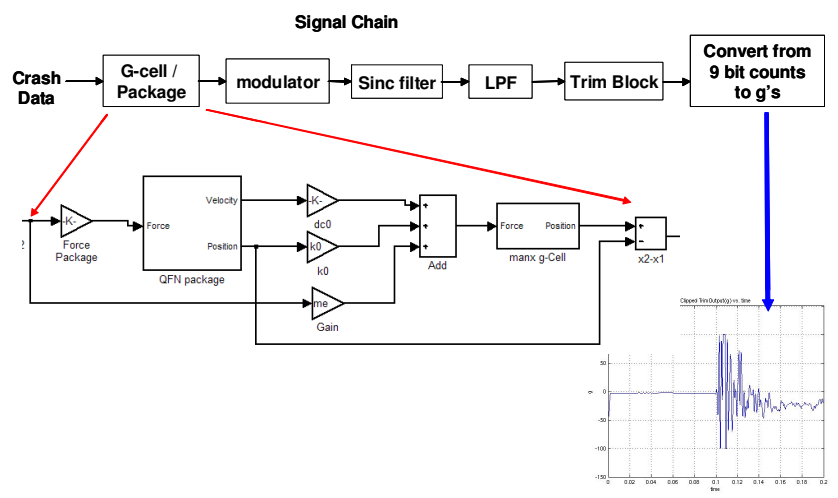

Figure 10: Microsystem model for crash simulation.

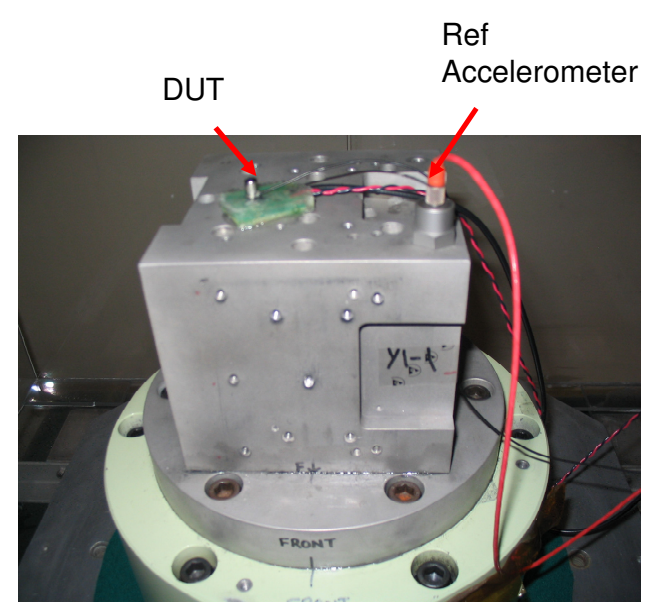

Figure 11: Experimenal setup to characterize sensor overload performance.

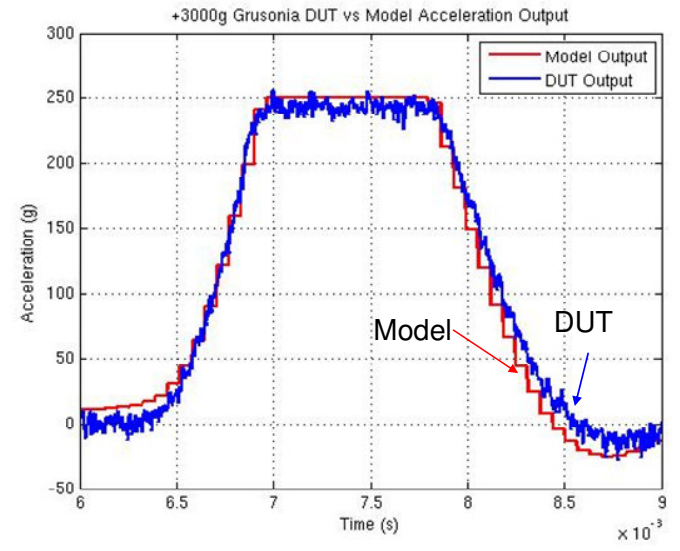

Figure 12: Comparison between overload microsystem simulation results and shock test data.

\section{CONCLUSIONS}

Nonlinear damping and higher order eigenmodes make significant contribution to the overload performance of MEMS inertial sensors. The nonlinear squeeze film damping is caused by the large gap change due to the large displacement of the proof mass under overload conditions. Higher order modes, when coupled with capacitance transfer function can cause nonlinear device output. It is shown that the microsystem model can accurately simulate the crash overload event when properly taking into accounts of these two sources of nonlinearity in MEMS dynamics.

\section{ACKNOWLEDGEMENTS}

The authors would like to thank Jan Menher, Torsten Hauck, Ilko Schmadlak, Patricia Ong and Lisa Zhang for insightful discussion and providing some of the experimental and simulation data.

\section{REFERENCES}

[1] M. Furmann and T. Miller, "Overload Performance of Inertial Sensors", Freescale Technical Enrichment Matrix Workshop, 06/26, Tempe, AZ (2007)

[2] J. J. Blech, "On Isothermal Squeeze Films", Journal of Lubrication technology, Vol. 105, pp 615-620 (1983).

[3] W. S. Griffin, et al., "A Study of Squeeze-film Damping”, J. of Basic Engineering, pp. 451-456 (1966)

[4] T. Veijola, "Equivalent Circuit Models for Micromechanical Inertial Sensors", Circuit Theory Laboratory Report Series CT-39, Helsinki Univ of Tech (1999)

[5] G. Schrag and G. Wachutka, "Accurate System-Level Damping Model for Highly Perforated Micromechanical devices", Sensors and Actuators A, 111, pp. 222-228 (2004)

[6] J. Mehner, L. Gabbay, S. Senturia, "Computer-Aided Generation of Nonlinear Reduced-Order Dynamic Macromodels", J. Microelectromech. Syst., vol. 9, pp. 269278 (2000).

[7] J. Mehner, et al., "The Influence of Packaging Technologies on the Performance of Inertial MEMS Sensors", the 15th International Conference on Solid-State Sensors, Actuators, and Microsystems, 06/21-25, pp. 1885-1888, Denver, CO (2009). 